\title{
Understanding Social Characteristic from Spatial Proximity in Mobile Social Network
}

\author{
D. Hu, B. Huang, L. Tu, S. Chen
}

\author{
Duan Hu,Benxiong Huang, \\ Lai Tu, Shu Chen* \\ Department of Electronics and Information Engineering \\ Huazhong University of Science and Technology \\ 1037 Luoyu Road, Wuhan, China \\ huduan1985@gmail.com, huangbx@hust.edu.cn, \\ laitu@hust.edu.cn, remark.chen@gmail.com \\ *Corresponding author: remark.chen@gmail.com
}

\begin{abstract}
Over the past decades, cities as gathering places of millions of people rapidly evolved in all aspects of population, society, and environments. As one recent trend, location-based social networking applications on mobile devices are becoming increasingly popular. Such mobile devices also become data repositories of massive human activities. Compared with sensing applications in traditional sensor network, Social sensing application in mobile social network, as in which all individuals are regarded as numerous sensors, would result in the fusion of mobile, social and sensor data. In particular, it has been observed that the fusion of these data can be a very powerful tool for series mining purposes. A clear knowledge about the interaction between individual mobility and social networks is essential for improving the existing individual activity model in this paper. We first propose a new measurement called geographic community for clustering spatial proximity in mobile social networks. A novel approach for detecting these geographic communities in mobile social networks has been proposed. Through developing a spatial proximity matrix, an improved symmetric nonnegative matrix factorization method (SNMF) is used to detect geographic communities in mobile social networks. By a real dataset containing thousands of mobile phone users in a provincial capital of China, the correlation between geographic community and common social properties of users have been tested. While exploring shared individual movement patterns, we propose a hybrid approach that utilizes spatial proximity and social proximity of individuals for mining network structure in mobile social networks. Several experimental results have been shown to verify the feasibility of this proposed hybrid approach based on the MIT dataset.
\end{abstract}

Keywords: Mobile social network; Geographic community; Community structure; Measurement;

\section{Introduction}

Recent years, with the increasing popularity of mobile terminal devices, the emerging massive urban sensors such as GPS, mobile phone and online user-generated social media enable us understand the intrinsic nature of human mobility such as high predictability in daily routine and the social demographic characteristics of the individuals. Given the importance of human mobility, e.g. human movement could be responsible for the geographical spread of human infectious diseases [2], many researchers have focused on capturing the statistical characteristics of individual human trajectories [3, 4], understanding the role of geographic distance and social interaction in human spatial redistribution on geographic scales[5-7]. Early empirical studies on albatrosses, monkeys and marine predators [8] suggested that animal trajectory can be approximated by a levy fight [9]. Given that money is carried by individuals, Brockmann [1] et al. illustrated individual geographic trajectory using half a million dollar bills, suggesting that 
human trajectories could be best modeled as a continuous-time random walk (CTRW model) with fat-tailed displacements and waiting-time distributions.

However, several types of empirical data [10]on human mobility, captured by mobile phone traces, show that the predictions of the CTRW models are systematically in conflict with the empirical results. Due to the fundamental importance of statistical properties of human travel, in recent years, the increasing pervasiveness of location-acquisition technologies such as $3 \mathrm{G}$ network and GPS is conducive to the collection of large spatial-temporal datasets and opening up new opportunities for discovering some different knowledge about movement behaviors. Indeed, the availability of these collected large-scale call data such as mobile phone records and global-positioning-system data, has offered researchers from various disciplines access to detailed patterns of human behavior, and greatly enhanced our understanding of human mobility [3]. Recently, distinguished from previous studies, a new understanding [4] about building individual predictive model for future locations suggests that at a global scale human mobility exhibits structural patterns subject to geographic and social constraints.

Researchers find that humans experience a combination of periodic movements limited by geographical factors and seemingly random jumps correlated with their social networks [4]. In this case, individual movements can be divided to the two parts: short-range travel driven by periodic movements and long-range travel driven by social interaction. Meanwhile, some studies $[4,5]$ found that individual (mobile devices) mobility patterns can shape and impact the inherent properties of social network. Similarity of individual mobility patterns, which is called "spatial proximity", is expected to exert a significant impact on the inner structure of such social networks, from the maintenance of long-lasting friendships to the formation of new links. It seems that social links are often driven by spatial proximity, from job- and family-imposed programs to joint involvement in various social activities [11]. On the contrary, the degree of similarity in periodic movements and social proximity of individuals can positively affect the level of spatial proximity since the similarity of both types of individual movements can be identified by spatial proximity. Based on collecting large-scale call data that are recorded, spatial proximity between any two mobile phone users has been used to predict the potential call in cell phone social networks [5].

The booming development of mobile social network gives rise to several amazing sub-issues, such as community detecting, link prediction, social-aware recommendation. This was explored through evaluating social property of individuals and mining network structure evolution using general social network analysis. However, given those fascinating features of spatial proximity for forming social interaction among individuals in mobile social network that has been revealed, it is just natural to consider the social influence of spatial proximity that can be used to improve current researches on those amazing sub-issues.

Therefore, the aim of this paper is to investigate the social features of spatial proximity and further mine social network structure evolution to explore some potential possibility for optimizing user experience of mobile social networking service and social recommendation in mobile social network. Although our present work is only confined to analyzing and applying the significant correlation between spatial proximity and social properties of individuals in mobile social network by several well-known data mining methods based on two real datasets, our experimental results also deepen our understanding about the social influence of spatial proximity in mobile social network.

The contributions of this paper are as follows:

- We propose a new measurement dimension called geographic community for clustering spatial proximity in mobile social network. A novel approach for detecting these geographic communities in mobile social network has been proposed. Through developing a spatial proximity matrix, an improved symmetric nonnegative matrix factorization method (SNMF)[13] is used to detect geographic communities in mobile social network. 
- We suppose that geographic community can provide a bridge between spatial proximity and social properties of individuals in mobile social network. By a real dataset containing thousands of mobile phone users in a provincial capital of China, the correlation between geographic community and common social properties of users has been shown.

- By mining shared individual movement patterns, we propose a hybrid approach that utilizes spatial proximity and social proximity of individuals for mining network structure in mobile social network. We first propose two new measurement matrixes. Combing these matrixes with the known adjacency matrix in social network, the joint nonnegative matrix factorization (JNMF) method has been used to mine network structure evolution. Finally, a collaborative matrix has been obtained to analyze the existing community structure and potential links in mobile social network.

The rest of this paper is organized as follows: section 2 describes a well-known index of spatial proximity. The two measurement matrixes for evaluating spatial proximity and social ties in such mobile social networks will also be formed. Section 3 presents the definition of our new measurement dimension "geographic community", and the strong correlation between geographic community and the similarity of several key indexes from the socio-economic background of those individuals based on thousands of cell phone call records and corresponding user information. Section 4 first provides a hybrid approach to mine network structure in mobile social network, and then show several experimental results to verify the feasibility of this proposed hybrid approach based on the MIT dataset. Section 5 provides related work and finally comes to the conclusion.

\section{Preliminaries}

In this section, we first choose spatial cosine similarity to represent the level of spatial proximity of any two individuals. The measurement matrixes that describe such spatial proximity of corresponding individuals have been presented. Furthermore, measurement matrixes that utilize the relationship between locations and users for evaluating spatial proximity and social ties in such mobile social networks have also been formed.

\section{Spatial cosine similarity[5]:SCos $(\mathrm{x}, \mathrm{y})$}

The cosine similarity of user $x$ and $y$ 's trajectories, describing how similar their visitation frequencies are, is represented by the cosine of the angle between the two vectors of number of visits at each location for $x$ and $y$ and,

$$
\operatorname{SCos}(x, y)=\sum_{l=L o c} \frac{P V(x, l) \times P V(y, l)}{\|P V(x, l)\| \times\|P V(y, l)\|} .
$$

Where $P V(x, l)=\sum_{i=1}^{n(x)} \delta\left(l, L_{i}(x)\right) / n(x)$ is the probability that user $x$ visits location $l, L o c$ is the set of all locations (cell phone towers and etc.).

\section{Spatial proximity matrix}

In order to reflect the spatial proximity between any two individuals in mobile social networks, we propose an approximate overlay $G=(V, E, w)$, then the adjacency matrix of $G$ can be written as: 


$$
G=\left(\begin{array}{ccc}
s_{11} & \cdots & s_{1 n} \\
\vdots & \ddots & \vdots \\
s_{n 1} & \cdots & s_{n n}
\end{array}\right)
$$

where $s_{i j}$ is spatial cosine similarity $S \operatorname{Cos}(i, j), i, j$ is each individual respectively. Specially, when $i=j, S \operatorname{Cos}(i, j)=1$. Note that $S \operatorname{Cos}(i, j)=S \operatorname{Cos}(j, i), G$ is a symmetric nonnegative matrix.

\section{The two measurement matrixes: user-location matrix and location-user matrix}

In previous studies, for evaluating the similarity in mobility patterns of two individuals, several indexes of spatial proximity have been proposed by the references [5, 14], spatial proximity can be defined by one key index of them:

The cosine similarity of user $x$ and $y$ 's and s trajectories, describing how similar their visitation frequencies are, is represented by the cosine of the angle between the two vectors of number of visits at each location for $x$ and $y$ :

$$
\operatorname{SCos}(x, y)=\sum_{l=L o c} \frac{P V(x, l) \times P V(y, l)}{\|P V(x, l)\| \times\|P V(y, l)\|} .
$$

Where $P V(x, l)=\sum_{i=1}^{n(x)} \delta\left(l, L_{i}(x)\right) / n(x)$ is the probability that user $x$ visits location $l, L o c$ is the set of all locations (cell phone towers and etc.).

As mentioned above, since the internal relationship between individuals and their usual travel locations can form spatial proximity, which has a significant impact on network structure of mobile social network, we develop two measurement matrixes that can reflect such internal relationship to evaluate network structure. Given $N$ individuals (or mobile personal devices) in a mobile social network and the number of all locations, we propose a user-location matrix $U$. (User-location matrix)

$$
U=\left(\begin{array}{ccc}
P V_{11} & \cdots & P V_{1 M} \\
\vdots & \ddots & \vdots \\
P V_{N 1} & \cdots & P V_{N M}
\end{array}\right)
$$

where $P V_{x l}=P V(x, l), x \leq N, l \leq M$

(Location-user matrix)

$$
L=\left(\begin{array}{ccc}
L V_{11} & \cdots & L V_{1 N} \\
\vdots & \ddots & \vdots \\
L V_{M 1} & \cdots & L V_{M N}
\end{array}\right)
$$

Where $L V_{x l}=\sum_{i=1}^{n(l)} \delta\left(l, L_{i}(x)\right) / n(x), x \leq N, l \leq M$ is the probability that user $x$ visits location $l$, $L O c$ is the set of all locations (cell phone towers and etc.). 


\section{Geographic community and its correlation with the social prop- erties of individuals}

In this section, we first propose a new measurement dimension for clustering those individuals with close spatial proximity in mobile social network. Based on SNMF method, an approach for detecting these geographic communities has been proposed. Several numerical experiments have been shown to verify the feasibility of this measurement approach.

\section{Geographic community}

Given $N$ individuals (mobile personal devices) in a mobile social network, the similar degree of geographic trajectories of two individuals can be measured by the spatial proximity index. In this case, we can develop an approximate overlay which indicates the spatial proximity between two individuals (nodes) in the mobile social network.

Suppose $G=(V, E, w)$ is an undirected weighted graph with $N$ nodes and $M$ links representing this approximate overlay. It is quite different from general social graphs in that these links between any two nodes are the spatial proximity index. The weight $w \in[0,1]$ of the edge $E$ denotes the similar degree of two individuals which can be calculated by spatial cosine similarity.

$C=C_{1}, C_{2}, \ldots, C_{k}$ denotes a collection of disjoint geographic communities, where $C_{i} \in C$ is a geographic community of $G$.

Using general definition of community structure in social network, the geographic community has several meanings: the nodes in geographic community are those individuals which have higher similar degree, that is, those individuals had met each other frequently in the past long period of time, unlike those individuals between different geographic communities. The strong correlation between this geographic similarity and their proximity in social network indicates that more social connections among the nodes will occur in geographic community in the future. Therefore, geographic community can be a measurement dimension that estimates the interaction between social community and spatial proximity. In fact, we consider geographic community as an indicator which may be used for evaluating the evolution of social community structure in the future.

\section{Detecting geographic community using symmetric nonnegative matrix factor- ization (SNMF)}

We consider symmetric NMF as our tool to find the geographic community because of its powerful interpretability and close relationship with clustering methods. It has been verified that SNMF has outstanding performance in detecting communities in undirected unweight complex networks.

Given that our approximate overlay is an undirected weighted virtual network, we further apply SNMF method to achieve our purpose. If $G$ is symmetric, then $S$ is symmetric. We can then absorb $S$ into $X$. i.e. $\hat{X}=X S^{1 / 2}$. Then our task is to solve the following problem:

$$
\min _{x \geq 0}\left\|G-\hat{X} \hat{X}^{T}\right\|_{F}^{2}
$$

According to [15], $\hat{X}$ can be obtained by the following multiplicative update rule

$$
\hat{X}_{i k} \leftarrow \hat{X}_{i k}\left[\frac{1}{2}+\frac{(G \hat{X})_{i k}}{\left(2 \hat{X} \hat{X}^{T} \hat{X}\right)_{i k}}\right]
$$




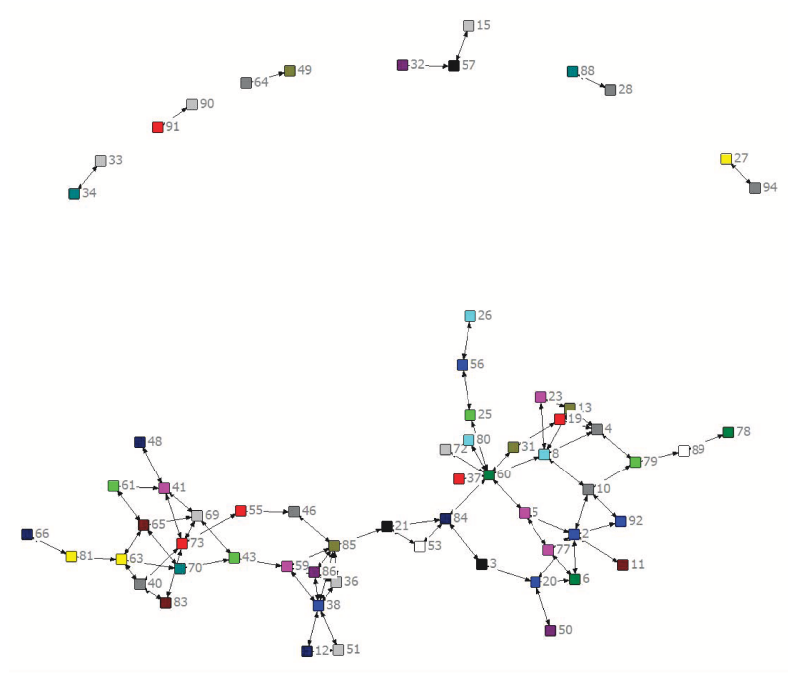

Figure 1: The reported friendship network in Reality Mining datasets

After iteration, the obtained $\hat{X}^{*}$ is just the scale partition matrix of the network $G$ of size $n \times K$, whose $i$-th row corresponds to the cluster (geographic community) membership of the $i$-th unit.

We can further normalize $\hat{X}$ to make $\sum_{j} \hat{X}_{i j}=1$ so that $\hat{X}_{i j}$ corresponds to the posterior probability that the $i$-th unit belongs to the $k$-th geographic community.

We choose Reality Mining data set [6] provided by the MIT Media Lab to test our proposed approach. The Reality Mining dataset contains communication, proximity, location, and activity information from 94 students or teachers at MIT during the 2004-2005 academic years. In particular, the dataset includes call logs, Bluetooth devices in proximity, cell tower IDs, application usage, and so on. In this dataset, a mobile social network which contains 94 nodes and several million links can be modeled as Fig.1, where the links representing real social friendship which can be evaluated by the number of calls, messages and so on.

To design this numerical experiment, we propose those cell tower ID lists of each individual communication record representing those locations 1 that can be used to calculate the probability $P V(x, l)$.We firstly obtain the spatial proximity matrix of this real mobile social network, then an approximate overlay network can be inferred in Fig.2. As is shown in Fig.2, this network does not have obvious community structure. We consider the reasons as follows: every two individuals have their spatial similarity. In the overlay network, the weights of links are inferred by corresponding spatial proximity. In this case, it is essential to properly evaluate the impact of the weight value on network structure. The binarization method will lose much useful information. Furthermore, these individuals have more overlaps in geographic community than general communities. Therefore, we choose to delete those weight links which have lower weights (i.e., the links that weight $w \leq 0.2$ will be deleted).

We use SNMF method for detecting geographic community on this overlay, the symmetric nonnegative matrix $G$ is a $n \times n(n=94)$ spatial proximity matrix. We assume the number of main geographic communities $K=2$, the $K \times n$ matrix will be calculated by (7). The final iteration result of $X$ is shown in Fig. 2.

In Fig. 3, the interval $[0,1]$ located in the Y-axis represents the community $I D=1$, the interval $[1,2]$ located in the $\mathrm{Y}$-axis represents the community $I D=2$, the sequence of nodes $i(i=1,2, \ldots, 94)$ is located in the $\mathrm{X}$-axis, and the gray level is obtained by corresponding element value of $X$, where the higher the gray level of the block $(i, I D(i))$ tend to be, the more 

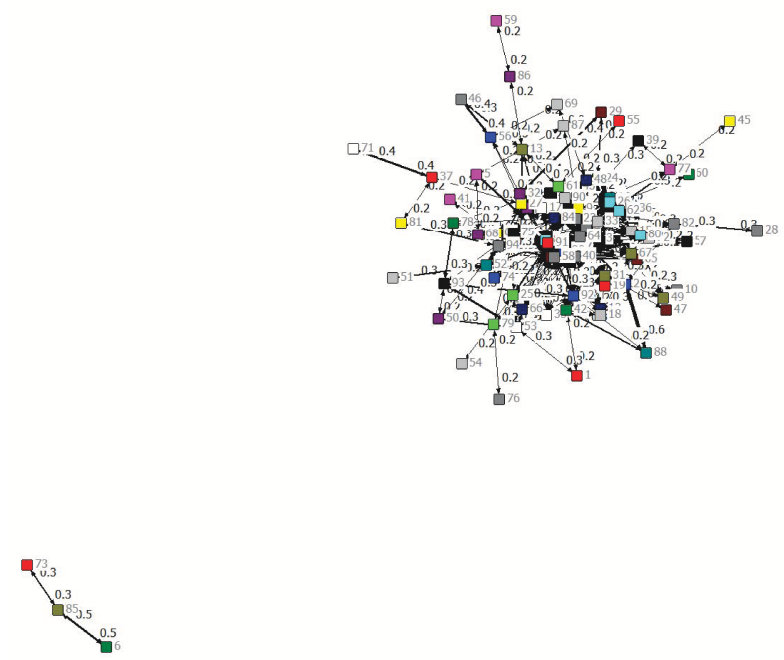

Figure 2: the spatial proximity overlay of reality mining datasets

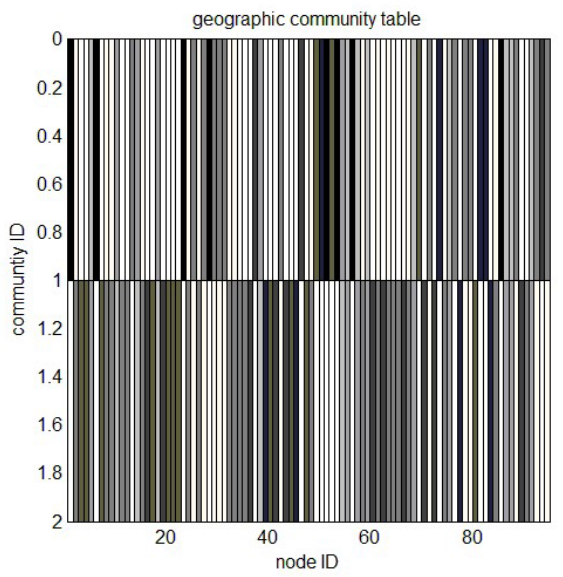

Figure 3: the results obtained by applying our proposed method 
likely that unit $i$ belongs to the geographic community $I D$.

\section{The relevance test between geographic community and the social properties of individuals}

We use a dataset that captures for two-month period the time-resolved trajectories of ten thousand anonymized mobile phone users in an anonymous provincial capital in China to uncover the relevance between geographic community and the social properties of individuals.

Given the multiplicity of the social properties of individuals and this dataset mainly contains the cell phone charging records of all users in an anonymous telecommunications company, the similarity of the cell phone charging of these users depends on the similarity of some social properties such as the economic ladder to some extent. Therefore, we choose the type of plan the user used and the interval of user charging per month as the two parameters of the economic ladder and present the value of the economic ladder of them. Three geographic communities have been obtained from ten thousand users by the above approach based on this dataset.

Through clustering these economic ladder values in each geographic community, we found that the economic ladder of individuals is highly consistent in each geographic community.

In this test, our parameters are set as follows:

Table 1: The initial situation of ten thousand mobile phone

\begin{tabular}{|c|c|c|}
\hline The number of individuals & Economic ladder & Matching results in general \\
\hline \multirow{3}{*}{10000} & $0($ budget $)(A R P U<100)$ & $5613(56.13 \%)$ \\
\cline { 2 - 3 } & $1($ ordinary $)(100<A R P U<200)$ & $3052(30.52 \%)$ \\
\cline { 2 - 3 } & $2($ luxury $)(A R P U>200)$ & $1335(13.35 \%)$ \\
\hline
\end{tabular}

Finally, our test truly shows the higher consistency in table2, given the number of geographic communities $N_{c}=3$, and we consider the posterior probability of nodes $\hat{X}_{i C}>0.4, C \in 0,1,2$ means node $i$ belong to $C$, then we obtained following numerical results:

Table 2: The matching results between the geographic communities and economic ladder

\begin{tabular}{|c|c|c|c|}
\hline The label of geographic communities & \multicolumn{3}{|c|}{ Matching results } \\
\hline 0(number $: 5767)$ & $63.4 \%$ & $28.85 \%$ & $7.6 \%$ \\
\hline 1 (number $: 4978)$ & $56.3 \%$ & $40.1 \%$ & $3.57 \%$ \\
\hline 2 (number $: 3244)$ & $44.1 \%$ & $35.2 \%$ & $20.6 \%$ \\
\hline
\end{tabular}

Given that periodic movements can explain about 50-70\% of the geographic trajectories of individuals, and the repetitive trajectories that are formed by periodic movement mainly depend on the role of daily routine and geographic features of human movement, we consider the similarity of the geographic trajectories between individuals is largely attributable to the overlapping functional areas for these individuals, for example, identical residential area, the same lunch venue and similar workplace. Furthermore, these overlapping functional areas just depend on the social properties of individuals, such as economic ladder, vocation, and so on. Therefore, the spatial proximity of individuals not only suggests the proximity of social relationship of individuals in mobile social network, but also implies to some extent the similarity of the social properties of individuals. Furthermore, we suggest that the similarity of the social properties of users also indicates the likeliness for these users to interact with each other. 


\section{A hybrid approach to mine network structure in mobile social network}

Since SNA techniques have gained extensive development in recent years, many studies on detecting existing community structure in various social networks can be found in an excellent survey [16]. Furthermore, a large amount of studies on predicting potential links have been presented for link prediction in complex networks [17]. Mining network structure, which provides us with meaningful insights into the internal structure of corresponding real social network and the possibilities to optimize the performance of social recommendation and link prediction, mainly contains two parts, i.e. detecting existing network structure and tracking the trend of network structure evolution. In general, mining network structure in mobile social networks has been investigated as a typical example of dynamic social networks by many researchers $[6,18]$.

Spatial proximity and social proximity of individuals can be used for mining network structure in mobile social network. Especially, existing community structure and potential social relationship in a real mobile social network can be obtained and analyzed by our hybrid approach. We first propose two new measurement matrixes which provide a bridge between spatial proximity and the social properties of individuals in mobile social networks. Combing these matrixes and the known adjacency matrix in social network, the joint nonnegative matrix factorization (JNMF) method [13] is used to mine network structure evolution. Finally, a collaborative matrix is obtained to analyze the existing community structure and potential links in mobile social network. Based on a real dataset, several experimental results have been shown to verify the feasibility of this proposed hybrid approach.

\section{Mining community structure in mobile social network using joint nonnegative matrix factorization (JNMF) method}

In previous studies, JNMF was usually used to detect community structure or hidden links in complex networks. In fact, it has been verified that JNMF has outstanding performance in such aspects in complex networks. In this section, we choose JNMF method as our tool to find community. Based on the MIT dataset, this social graph is an undirected weight network. Given its adjacency matrix of friend graph D, we further apply JNMF method to achieve our purpose. The problem we aim to solve is

$$
\begin{aligned}
& \min _{x \geq 0} l(X, U, D, L) \\
& \text { s.t. } X \in \mathbb{R}_{+}^{n \times m}
\end{aligned}
$$

Where $l(X, U, D, L)^{\text {def }}=\|U-X\|^{2}+\alpha\left\|D-X X^{T}\right\|^{2}+\beta\left\|L-X^{T} X\right\|^{2}$, and $\alpha>0, \beta>0$ are constants to tradeoff the importance between different terms. Given $N$ individuals (or mobile personal devices) in a mobile social network, and the number of all locations $m$, and $D$ represents the adjacency matrix of friend graph , $U$ and $L$ represent User-location matrix (4)and locationuser matrix (5), respectively . According to [15], $\hat{X}$ can be solved by the following multiplicative update rule.

$$
\hat{X}_{i j} \leftarrow \hat{X}_{i j}\left[\frac{[U+2 \alpha D X+X \hat{L}]_{i j}}{2(\alpha+\beta)\left[X X^{T} X\right]_{i j}}\right]^{\frac{1}{4}}
$$

where $\hat{D}=2 \beta D-1$

After iteration, the obtained $\hat{X}^{*}$ is just the scale partition matrix of the network $G$ of size $N \times M$, and then we obtain the hybrid matrix $C=X^{T} X$. Let this matrix is the initial adjacent matrix, we further use the above approach in section 3 for detecting community structure based on SNMF. 


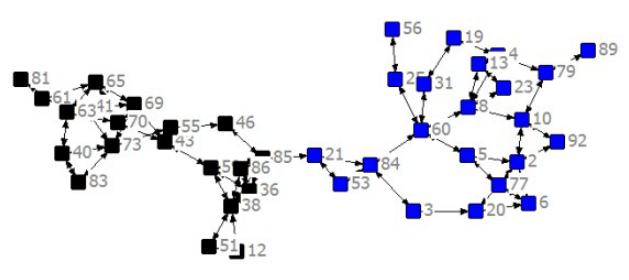

Figure 4: Two main communities in the community structure of this friendship network

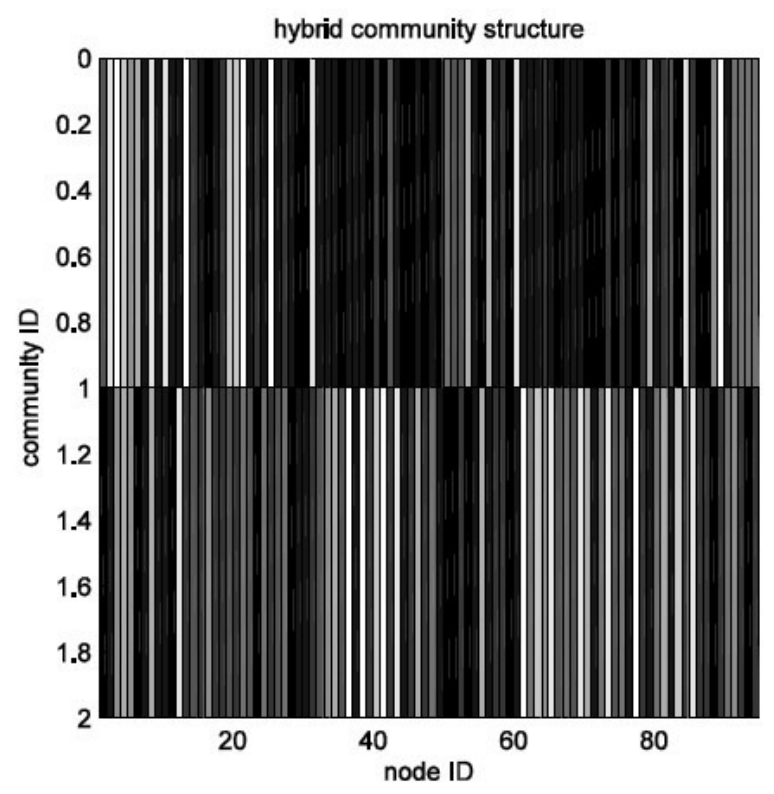

Figure 5: The hybrid communities structure in this MIT dataset

In our experiment, we also use Reality Mining data set [6] provided by the MIT Media Lab to test our proposed approach. From this real friendship network, two main communities can be inferred by real social connections as shown in Fig.2, which shows the first-year business school students and individuals working together in the same building respectively. Moreover, we can simply detect these social communities by Newman method. However, the dynamic evolution of the social ties among these individuals cannot be timely detected. This leaves an important question that to what extent individual mobility patterns impacts the social network. Therefore, in this section, the communities of the same dataset will be detected by our proposed method.

During our implementation process, we suppose spatial proximity effect on social community structure is limited, and present two weight parameters. As is shown in Fig.5, the difference in gray-level between two communities labels for nodes illustrates the segmentation map presented in Fig.4. Meanwhile, some nodes (ex: $i=2,33,76)$ that are not covered by this segmentation map shows the potential possibility for joining the two communities together.

In Fig. 5, the interval $[0,1]$ located in the Y-axis represents the community $I D=1$,and the interval $[1,2]$ located in the Y-axis represents the community $I D=2$, the sequence of nodes $i(i=1,294)$ is located in the $\mathrm{X}$-axis, and the gray level is obtained by corresponding element 
value of $X$, where the higher the gray level of the block $(i, I D(i))$ tend to black, the more likely that the unit $i$ belongs to the geographic community $I D$.

\section{Results and Discussion}

Since recent research progress on the interaction between human mobility and social ties has provided us with valuable information that spatial proximity can be used to predict future social links, in this paper, we first proposed a new measurement dimension (geographic community) for evaluating the spatial proximity influence on the evolution of social community structure in mobile social networks.

The correlation between geographic community and common social properties of users has been analyzed based on a real dataset. We found the spatial proximity of individuals not only suggests the proximity of social relationship of individuals in mobile social networks [5], but also implies to some extent the similarity of the social properties of individuals. Then a hybrid approach that utilizes spatial proximity and social homophily of individuals for mining network structure in mobile social network has been proposed.

However, due to the inner ambiguity of individuals spatial proximity, the direct applicability of spatial proximity in mobile social network still leaves much room to be studied. The results presented in this paper will lead us to two interesting directions for future research. The first direction is to explore the common social properties of individuals based on those common functional areas that can be obtained from spatial proximity, since we consider high spatial proximity should be driven by similar social feature of individuals to a certain extent and even develop a hybrid technique for personalized recommendation utilizing geographic locations and social network. The second direction is to develop a routing algorithm on MANET (mobile ad-hoc network) using the inherent property of geographic community, since this communication services that rely on this type of data transfer will strongly depend on human mobility characteristics and how often such transfer arises.

\section{Bibliography}

[1] D. Brockmann, L. Hufnagel, and T. Geisel (2006); The scaling laws of human travel, Nature, 439:462-465.

[2] L. Hufnagel, D. Brockmann, and T. Geisel (2004); Forecast and control of epidemics in a globalized world, Proceedings of the National Academy of Sciences of the United States of America, 101(42):15124-15129.

[3] C. Song, Z. Qu, N. Blumm, A. Barabasi (2010); Limits of predictability in human mobility, Science, 327(5968): 1018-1021.

[4] E. Cho, S. A. Myers, and S. J. Leskovec(2011); Friendship and mobility: User movement in location-based social networks, Proc. of the 17th ACM SIGKDD international conference on Knowledge discovery and data mining, New York, USA, 1082-1090.

[5] D. Wang, D. Pedreschi, C. Song, F. Giannotti, and A. Barabasi (2011); Human mobility, social ties, and link prediction, Proc. of the 17th ACM SIGKDD international conference on Knowledge discovery and data mining, New York, USA, 1100-1108.

[6] N. Eagle, A. Pentland, and D. Lazer (2009);Inferring friendship network structure by using mobile phone data, Proc. of the National Academy of Sciences, 106(36): 15274-15278. 
[7] L. Backstrom, E. Sun, and C. Marlow (2010); Find me if you can: improving geographical prediction with social and spatial proximity, Proc. of the 19th international conference on World wide web(WWW'10), New York, USA, 61-70.

[8] M. C. Gonzalez, C. A.Hidalgo, and A. Barabasi (2008); Understanding individual human mobility patterns, Nature, 453: 779-782.

[9] R. N. Mantegna and H. E. Stanley (1994); Stochastic process with ultraslow convergence to a Gaussian: the truncated Levy flight, Physical Review Letters, 73: 2946-2949.

[10] C. Song, T. Koren, and A. Barabasi (2010); Modelling the scaling properties of human mobility, Nature Physics, 6: 818-823.

[11] M. T. Rivera, S. B. Soderstrom, and B. Uzzi (2010); Dynamics of dyads in social networks: Assortative, relational, and proximity mechanisms, Annual Review of Sociology, 36: 91-115.

[12] Q. Hao, et al. (2010); Equip tourists with knowledge mined from travelogues, Proceedings of the 19th international conference on World wide web (WWW'10), New York, USA, 401-410.

[13] F. Wang, et al. (2011); Community discovery using nonnegative matrix factorization, Data Mining and Knowledge Discovery, 22: 493-521.

[14] Q. Li, et al. (2008); Mining user similarity based on location history, Proceedings of the 16th ACM SIGSPATIAL international conference on Advances in geographic information systems(GIS'08), Irvine, CA, USA, 34-43.

[15] D. Wang, T. Li, S. Zhu, and C. Ding (2008); Multi-document summarization via sentencelevel semantic analysis and symmetric matrix factorization, Proc. of the 31st annual international ACM SIGIR conference on Research and development in information retrieval(SIGIR'08 ), New York, NY, 307-314.

[16] S. Fortunato (2010); Community detection in graphs, Physics Reports, 486: 75-174.

[17] L. Lu and T. Zhou (2011); Link prediction in complex networks: A survey, Physica A: Statistical Mechanics and its Applications, 390: 1150-1170.

[18] N. P. Nguyen, et al. (2011); Adaptive algorithms for detecting community structure in dynamic social networks, Proc.IEEE INFOCOM, Shanghai,China, 2282-2290. 